\title{
Role of Bone Grafts in Implant Surgery: A Review
}

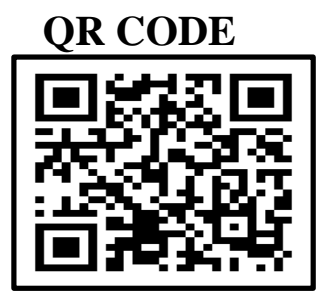

\section{CHANDNI ${ }^{10}$, VINIT KUMAR*2}

Introduction of dental implants and more concern in patients about esthetics after loss of natural teeth increases the demand of bone grafts. Various types of bone grafts as allografts, xenografts and autografts are used all with their own pros and cons. Bone grafts are more likely to succeed when the conditions at the recipient site are favourable and certain requirements are fulfilled. This review explores the use of various bone grafts in implant dentistry.

KEYWORDS: Bone Grafts, Augmentation, Bone defects, Implants

\section{INTRODUCTION}

The replacement of missing teeth by osseointegrated dental implants is a commonly utilized treatment option in dentistry. But results are dependent upon the type of bone at the recipient site, the site depends upon the bone generation process. As a result, the bone grafts are applied which changes the biological response into a regenerative rather than a predominant reparative pattern of healing. Use of guided tissue regeneration membranes increases the clinical success by providing better protection and containment of bone substitute in the defect.

\section{BONE AUGMENTATION PROCEDURES:}

Bone graft materials are defined as any synthetic materials that are available as bone grafts. These are safe materials. New bone is formed through osteogenic, osteoinductive or osteoconductive processes. However, these three processes have a difference in their mechanism of new bone formation.

Various types of augmentation procedures are available as:
1. AUTOGRAFTS
2. ALLOGRAFTS
3. XENOGRAFTS

These grafts act as scaffold which promotes new bone formation by osteogenesis, osteoconduction and osteoinduction, which are described as follows:

Osteogenesis: This process allows new bone formation by the action of osteoprogenitor cells that are present in the bone grafts.

Osteoconduction: Here, the graft itself functions as a scaffold upon which osteogenic cells move from the adjacent bone margins, to proliferate and allow new bone formation with replacement of the graft material with new bone. ${ }^{1}$

Osteoinduction: It leads to bone fill by the mechanism of transformation of undifferentiated mesenchymal stem cells from the tissue surrounding the graft to differentiate into osteogenic cells till new bone formation occurs. ${ }^{2}$

As a result, the treatment results are dependant upon the type of bone and sufficient bone quantity at the site of surgery for future implant treatment. ${ }^{3}$

When planning augmentation procedures of bone defects i.e. cases of bone deficient sites where Implant placement is difficult, particularly of the maxillary sinus or guided bone regeneration procedures of alveolar ridge, different types of bone graft materials play their role. In these situations it is impossible to place implants with bone grafts only. ${ }^{4}$

\section{BONE GRAFT MATERIALS}

Ideal graft material should have a resorption rate similar to the rate of new bone formation, resulting in an augmented site that consists of host bone alone.

(C) Chandni et al. This is an open access article distributed under the terms of the Creative Commons Attribution License CC-BY-NC 4.0, which permits unrestricted use, distribution and reproduction in any medium, provided the use is not commercial and the original author(s) and source are cited. Submitted on: 18-Aug-2021; Accepted on: 03-Nov-2021 
The ideal bone graft material should be:

1. Biocompatible: Ideally a bone Graft should be atleast identical to the bone in its physical and chemical structure leading to angiogenesis and fibrovascular tissue ingrowth at the desired site/surgical site.

2. Osteogenic or osteoinductive: It should be osteoconductive and leads to new bone formation and growth along the graft material.

3. Remodeling: The speed of remodelling of a graft material should be such that the rate of resorption is equal to the rate of formation of new bone.

\section{BONE GRAFTS FOR IMPLANT DENTISTRY}

In the present scenario, an Implant procedure is a treatment that replaces missing teeth, improves function, and enhances esthetics. However, cases of bone deficient sites where Implant placement is difficult, bone grafts play their role. In these situations, it is possible to place Implants with bone grafts only.

\section{THE RATIONALE FOR BONE GRAFTS}

Implant placement requires sufficient volume of bone and bone quality in a biological way. This is achieved by the particular implant design, which requires certain dimensional properties that is long lasting success.

Indications for Bone Grafts in Implant treatment are:

- In alveolar sockets after extraction

- Refilling a local bony defect due to trauma or infection

- Refilling a peri-implant defect due to periimplantitis

- Vertical augmentation procedures of the mandible and maxilla

- Horizontal augmentation in the mandible and maxilla

\section{RIDGE PRESERVATION TECHNIQUE}

The techniques are beneficial as they decrease horizontal and vertical bone loss after extraction versus healing by blood clot alone. After extraction of natural teeth, in the first 2 years $40-50 \%$ of height and width of bone are lost, which hampers the alignment of Implant and their function as well as their esthetics. To avoid these side effects various techniques of ridge preservation are used to decrease horizontal and vertical bone loss after extraction.
The aims of ridge preservation techniques are:

- To avoid horizontal and vertical loss of bone

- Prevent soft tissue loss

- Avoid Alveolar ridge volume loss

- To avoid augmentation procedures or costly grafts.

\section{BONE GRAFT CLASSIFICATION BY MATERIAL SOURCE}

Many techniques are available to horizontally augment the alveolar ridge effectively and with good prediction.

Vertical augmentation techniques: These are not as effective and are not used for augmentation as compared to horizontal augmentation and lead to more complications. ${ }^{5}$ Bone grafts lead to success when there are favourable conditions at the recipient site and there are certain requirements.

Four different types of bone graft materials have been commonly used and are classified as: Autografts, allografts, xenografts and alloplasts.

1. Allografts: Allografts are type of grafts that are prepared from bone matrix granules that are demineralized and these have different shapes.The granules are placed and bone is formed in the areas where planning of Implants have been done in the near future. These are indicated and are an effective alternative to autografts.

When large areas are indicated for grafting, an autogenous bone in the form of shell is used often as a biologic container. Then these grafts act as a scaffold on which new bone is formed as cells of graft die after few days and the remaining graft acts as a resorbable membrane.

In autogenous bone the cells present as bone cells die after a few days and then the bone plate functions as a stable, avital, a slowly resorbable membrane. Autogenous bone has both advantages and disadvantages which can be summarized as follows:

Autogenous bone must be harvested from certain intraoral or extraoral sites, with a comparitively higher morbidity as compared to nonautogenous materials (ie, risk of neural disturbances in case of intraoral grafts due to possible lesions of the inferior alveolar nerve branches, and gait disturbances in case 
of harvesting from the iliac crest).

When a delayed implant treatment is planned, maxillary sinuses grafted with autogenous bone may receive implants in an earlier way as compared to non-autogenous bone substitutes. ${ }^{2}$

When sinus grafting procedures are planned, Autogenous bone is the material of choice and an associated onlay grafting of the maxilla is planned in the case of severe atrophy. In converse, there is a lack of information regarding such reconstructions with non-autogenous materials.

2. Allogenic bone blocks: The allogenic bone blocks in the form of blocks can also be used for a shell technique which acts as a substitute for an autogenous bone. ${ }^{6}$

Different bone grafting materials like autogenous, allogenic, xenogenic or alloplastic materials are used to fill the space between the local bone and surrounding shell.

3. Xenograft: It is derived from bone of another species (bovine or cow). It is processed at a very high temperature to avoid immune rejection and contamination. ${ }^{5}$ It also acts as a scaffold on which new bone is formed from surrounding bone. This graft is osteoconductive and availability is present in various shapes and sizes. The greatest advantage of this graft is that there is no requirement of any second surgery to harvest graft from own body. Bovine collagen can also be used. It is produced by mixing untreated collagen and heat denatured collagen which is then freeze dried and then cross linked. Then sponge blocks are formed to place into the socket. Studies show increased healing with this xenograft in the extraction socket. ${ }^{5}$

4. Alloplast: These synthetic materials are available that act as bone grafts. These are safe materials. The most commonly used alloplastic materials are calcium phosphate based ceramics such as hydroxyapatite and tricalcium phosphate.

Tricalcium phosphates: The bio-active and materials that resorb are calcium phosphates. Bone cells proliferate and attach to the host cells. The graft is then removed from the implant site as bone grows into the formed scaffold. Initially there is an attachment of the graft into the matrix of bone and then degradation process occurs in a gradual way.

Hydroxyapatite: Hydroxyapatite ceramics when used as bone grafts are almost identical to natural bone in composition. Natural bone is made up of $2 / 3^{\text {rd }}$ hydroxyapatite which is the inorganic part of the bone. It remains at the recipient site for a longer period due to its low solubility. It is an excellent biodelivery vehicle for growth factors and osteogenic cell population.5 Grafts made up of Tricalcium phosphate alone are completely resorbed and they are replaced with bone within 5-15 months. As time passes full absorption of graft occurs and then replacement with bone occurs.

The basic principle of using HA and TCP in combination is a balance between the stable HA which can be found years after implantation, and the fast resorbing Tricalcium phosphate. The ratio between the two affects the resorptive properties of the graft material. A ratio between 65:35 and 55:45 of HA to TCP has been proven particularly suitable in many studies. ${ }^{7}$ The HA remains at the recipient site for a longer period due to its low solubility. It is an excellent biodelivery vehicle for growth factors and osteogenic cell population. Products with TCP alone are completely resorbed and replaced by bone within five to 15 months. ${ }^{2}$

Bioactive glass: It is a synthetic graft material. There is an extensive use of this graft. It is a reactive graft when compared to inert materials like HA or TCP. It allows new bone formation by the release of mineral ions, that contributes to good properties of the graft.

After mixing with blood, the graft binds with bone and there is release of silica ions. These ions allow differentiation and proliferation of osteoblasts. With time, there is complete absorption of the graft and replacement with bone occurs.

Consensus on Surgical Techniques and Materials

- The survival of implants placed into grafted areas when compared with survival rates of implants placed into natural bone. ${ }^{3}$

The quality of the bone allows placement of graft material at the recipient site of the graft. The cancellous bone is preferred over cortical bone at the recipient site of the grafts. 
The cells within the cancellous bone contribute to 60 percent of the healing capacity of the bone. In a young, healthy patient the periosteum contributes to 30 percent of the total healing capacity.

Cells in the cortical bone allows only to percent of bone healing. After extraction, when bone resorbs, cancellous bone shrinks more in comparison to cortical bone. As the cancellous compartment of the bone decreases, the provision for osteoblasts also decreases. Computerized tomography can reveal the ratio of cancellous bone to cortical at the recipient site prior to surgery.

This ratio between cancellous: cortical bone helps in the selection of the graft material as follows:

1. Only cortical bone-autograft is used.

2. Cortico-cancellous-depends on predominant type

3. Mostly cancellous-everything is possible. ${ }^{8}$

Ridge preservation techniques are beneficial as these decreases horizontal and vertical bone loss after extraction versus healing by blood clot alone.

The ridge preservation techniques help in maintaining ridge width and height. Most of the graft materials are used in these techniques and these are effective with only slight differences between them.

'External' augmentation procedures, both horizontal and vertical, on the alveolar ridge are more difficult than 'internal' augmentation procedures in sites like the maxillary sinus. ${ }^{3}$

In comparison, augmentation of vertical alveolar ridge defects have higher complications than those for horizontal defects. ${ }^{5}$ And lastly, the prognosis gets worsened by blood supply, trauma or extensive surgery in the area, in smokers. ${ }^{5}$

Uncontrolled diabetes, radiation to head and neck, bisphosphonate therapy are at least relative contraindications for bone augmentation. ${ }^{6}$

Choice of grafting material: Grafting materials other than autogenous grafts are preferable for sinus floor elevation, with similar results in clinical outcomes and implant survival. ${ }^{9}$

\section{CONCLUSION}

Current literature shows that different bone graft as well as non-grafting treatment options are available for implant procedure each with their own advantages and disadvantages. Till today, we can restore a fraction of the bone volume in extent due to structural and interactive complexity of periodontium. Future research and new techniques, materials and advances in genetics, molecular biology, cell biology and biomaterials, have opened the door for new regenerative techniques based upon the recent development approach. Preference is given to less invasive procedures that carry lower risk of complications.

\section{REFERENCES}

1. Sheikh Z, Sima C, Glogauer M. Bone Replacement Materials and Techniques Used for Achieving Vertical Alveolar Bone Augmentation. Materials (Basel). 2015;8(6):2953-93. https://doi.org/10.3390/ma8o62953. 2. Starch-Jensen T, Deluiz D, Vitenson J, Bruun NH, Tinoco EMB. Maxillary Sinus Floor Augmentation with Autogenous Bone Graft Compared with a Composite Grafting Material or Bone Substitute Alone: a Systematic Review and Meta-Analysis Assessing Volumetric Stability of the Grafting Material. J Oral Maxillofac Res. 2021 Mar 31;12(1):e1. https://doi.org/10.5037/jomr.2021.12101.

3. Buser D, Chappuis V, Belser CU, Chen S. Implant placement post extraction in esthetic single tooth sites: when immediate, when early, when late. Periodontol 2000. 2017;73(1):84-102. https://doi.org/10.1111/prd.12170. 4. Khojasteh A, Kheiri L, Motamedian SR, Khoshkam V. Guided Bone Regeneration for the Reconstruction of Alveolar Bone Defects. Ann Maxillofac Surg. 2017;7(2):263-77. https://doi.org/10.4103/ams.ams_76_17. 5. Goldstep F. Bone Grafts For Implant Dentistry: The Basics. (Online Article). Available from: https://www.oralhealthgroup.com/features/100391836o/ [Last Accessed on 15th July, 2021]

6. Gomes KU, Carlini JL, Biron C, Rapoport A, Dedivitis RA. Use of allogeneic bone graft in maxillary reconstruction for installation of dental implants. J Oral Maxillofac Surg. 2008;66(11):2335-8. https://doi.org/10.1016/j.joms.2008.06.006.

7. McAllister BS, Haghighat K. Bone augmentation techniques. J Periodontol. 2007;78(3):377-96. https://doi.org/10.1902/jop.2007.060048

8. Klein MO, Al-Nawas B. For Which Clinical Indications In Dental Implantology Is The Use Of Bone Substitute Materials Scientifically Substantiated? Eur J Oral Implantol. 2011; 4 (suppl): S11-S29

9. Bauer TW, Muschler GF. Bone graft materials. An 
overview of the basic science. Clin Orthop Relat Res.

2000

;(371):10-27.

Source of support: Nil, Conflict of interest: None declared

Cite this article as:

Chandni, Kumar V. Role of Bone Grafts in Implant Surgery: A Review. Int Healthc Res J. 2021;5(8):RV1-RV5. https://doi.org/10.26440/IHRJ/0508.11464

AUTHOR AFFILIATIONS: ( ${ }^{*}$ Corresponding Author)

1. Department of Periodontology and Oral Implantology (https://orcid.org/oooo-0002-8820-0204)

2. Department of Oral and Maxillofacial Surgery

Desh Bhagat Dental College and Hospital, Mandi Gobindgarh

Contact Corresponding Author At: dr.vinitkathpalmds[at]gmail[dot]com 